Research Article

\title{
Permanence of a Discrete $n$-Species Schoener Competition System with Time Delays and Feedback Controls
}

\section{Xuepeng Li and Wensheng Yang}

School of Mathematics and Computer Science, Fujian Normal University, Fuzhou 350007, China

Correspondence should be addressed to Wensheng Yang, ywensheng@126.com

Received 4 March 2009; Revised 25 July 2009; Accepted 3 September 2009

Recommended by John Graef

A discrete $n$-species Schoener competition system with time delays and feedback controls is proposed. By applying the comparison theorem of difference equation, sufficient conditions are obtained for the permanence of the system.

Copyright (C) 2009 X. Li and W. Yang. This is an open access article distributed under the Creative Commons Attribution License, which permits unrestricted use, distribution, and reproduction in any medium, provided the original work is properly cited.

\section{Introduction}

In 1974, Schoener [1] proposed the following competition model:

$$
\begin{aligned}
& \dot{x}=r_{1} x\left(\frac{I_{1}}{x+e_{1}}-r_{11} x-r_{12} y-c_{1}\right), \\
& \dot{y}=r_{2} y\left(\frac{I_{2}}{y+e_{2}}-r_{21} x-r_{22} y-c_{2}\right),
\end{aligned}
$$

where $r_{i}, I_{i}, e_{i}, r_{i j}, c_{i}(i=1,2 ; j=1,2)$ are all positive constants.

May [2] suggested the following set of equations to describe a pair of mutualists:

$$
\begin{aligned}
& \dot{u}=r_{1} u\left(1-\frac{u}{a_{1}+b_{1} v}-c_{1} u\right), \\
& \dot{v}=r_{2} v\left(1-\frac{v}{a_{2}+b_{2} u}-c_{2} v\right),
\end{aligned}
$$


where $u, v$ are the densities of the species $U, V$ at time $t$, respectively. $r_{i}, a_{i}, b_{i}, c_{i}, i=1,2$ are positive constants. He showed that system (1.2) has a globally asymptotically stable equilibrium point in the region $u>0, v>0$.

Both of the above-mentioned works are considered the continuous cases. However, many authors [3-5] have argued that the discrete time models governed by difference equations are more appropriate than the continuous ones when the populations have nonoverlapping generations. Bai et al. [6] argued that the discrete case of cooperative system is more appropriate, and they proposed the following system:

$$
\begin{aligned}
& x_{1}(k+1)=x_{1}(k) \exp \left\{r_{1}(k)\left[1-\frac{x_{1}(k)}{a_{1}(k)+b_{1}(k) x_{2}(k)}-c_{1}(k) x_{1}(k)\right]\right\} \\
& x_{2}(k+1)=x_{2}(k) \exp \left\{r_{2}(k)\left[1-\frac{x_{2}(k)}{a_{2}(k)+b_{2}(k) x_{2}(k)}-c_{2}(k) x_{1}(k)\right]\right\} .
\end{aligned}
$$

On the other hand, as was pointed out by Huo and Li [7], ecosystem in the real world is continuously disturbed by unpredictable forces which can result in changes in the biological parameters such as survival rates. Practical interest in ecology is the question of whether or not an ecosystem can withstand those unpredictable disturbances which persist for a finite period of time. In the language of control variables, we call the disturbance functions as control variables. During the last decade, many scholars did excellent works on the feedback control ecosystems (see [8-11] and the references cited therein).

Chen [11] considered the permanence of the following nonautonomous discrete Nspecies cooperation system with time delays and feedback controls of the form

$$
\begin{gathered}
x_{i}(k+1)=x_{i}(k) \exp \left\{r_{i}(k)\left[1-\frac{x_{i}\left(k-\tau_{i i}\right)}{a_{i}(k)+\sum_{j=1, j \neq i}^{n} b_{i j}(k) x_{j}\left(k-\tau_{i j}\right)}-c_{i}(k) x_{i}\left(k-\tau_{i i}\right)\right]\right. \\
\left.-d_{i}(k) \mu_{i}(k)-e_{i}(k) \mu_{i}\left(k-\eta_{i}\right)\right\}, \\
\Delta \mu_{i}(k)=-\alpha_{i}(k) \mu_{i}(k)+\beta_{i}(k) x_{i}(k)+\gamma_{i}(k) x_{i}\left(k-\sigma_{i}\right),
\end{gathered}
$$

where $x_{i}(k)(i=1, \ldots, n)$ is the density of cooperation species $X_{i}, \mu_{i}(k)(i=1, \ldots, n)$ is the control variable ([11] and the references cited therein).

Motivated by the above question, we consider the following discrete $n$-species Schoener competition system with time delays and feedback controls:

$$
\begin{gathered}
x_{i}(k+1)=x_{i}(k) \exp \left\{\frac{r_{i}(k)}{x_{i}\left(k-\tau_{i}\right)+a_{i}(k)}-\sum_{j=1}^{n} b_{i j}(k) x_{j}\left(k-\tau_{j}\right)-c_{i}(k)\right. \\
\left.-d_{i}(k) \mu_{i}(k)-e_{i}(k) \mu_{i}\left(k-\eta_{i}\right)\right\}, \\
\Delta \mu_{i}(k)=-\alpha_{i}(k) \mu_{i}(k)+\beta_{i}(k) x_{i}(k)+\gamma_{i}(k) x_{i}\left(k-\sigma_{i}\right),
\end{gathered}
$$


where $x_{i}(k)(i=1,2, \ldots, n)$ is the density of competitive species at $k$ th generation; $\mu_{i}(k)$ is the control variable; $\Delta$ is the first-order forward difference operator $\Delta \mu_{i}(k)=\mu_{i}(k+1)-\mu_{i}(k), i=$ $1,2, \ldots, n$.

Throughout this paper, we assume the following.

$\left(H_{1}\right) \alpha_{i}(k), \beta_{i}(k), \gamma_{i}(k), a_{i}(k), b_{i j}(k), r_{i}(k), c_{i}(k), d_{i}(k), e_{i}(k), i=1,2, \ldots, n$ are all bounded nonnegative sequence such that

$$
\begin{gathered}
0<\alpha_{i}^{l} \leq \alpha_{i}^{u}<1, \quad 0<\beta_{i}^{l} \leq \beta_{i}^{u}, \quad 0<r_{i}^{l} \leq r_{i}^{u}, \quad 0<a_{i}^{l} \leq a_{i}^{u}, \\
0<b_{i j}^{l} \leq b_{i j}^{u}, \quad 0<r_{i}^{l} \leq r_{i}^{u}, \quad 0<c_{i}^{l} \leq c_{i}^{u}, \quad 0<d_{i}^{l} \leq d_{i}^{u}, \quad 0<e_{i}^{l} \leq e_{i}^{u} .
\end{gathered}
$$

Here, for any bounded sequence $\{a(k)\}, a^{u}=\sup _{k \in N} a(k), a^{l}=\inf _{k \in N} a(k)$.

$\left(H_{2}\right) \tau_{i}, \eta_{i}, \sigma_{i}, i=1, \ldots, n$ are all nonnegative integers.

Let $\tau=\max \left\{\tau_{i}, \eta_{i}, \sigma_{i}, i=1, \ldots, n\right\}$, we consider (1.5) together with the following initial conditions:

$$
\begin{array}{lll}
x_{i}(\theta)=\varphi_{i}(\theta), & \theta \in N[-\tau, 0]=\{-\tau,-\tau+1, \ldots, 0\}, & \varphi_{i}(0)>0, \\
\mu_{i}(\theta)=\phi_{i}(\theta), & \theta \in N[-\tau, 0]=\{-\tau,-\tau+1, \ldots, 0\}, & \phi_{i}(0)>0 .
\end{array}
$$

It is not difficult to see that solutions of (1.5) and (1.7) are well defined for all $k \geq 0$ and satisfy

$$
x_{i}(k)>0, \quad \mu_{i}(k)>0 \quad \text { for } k \in Z, i=1,2, \ldots, n \text {. }
$$

The aim of this paper is, by applying the comparison theorem of difference equation, to obtain a set of sufficient conditions which guarantee the permanence of the system (1.5).

\section{Permanence}

In this section, we establish a permanence result for system (1.5).

Definition 2.1. System (1.5) is said to be permanent if there exist positive constants $M$ and $m$ such that

$$
\begin{aligned}
& m \leq \lim _{k \rightarrow+\infty} \inf x_{i}(k) \leq \lim _{k \rightarrow+\infty} \sup x_{i}(k) \leq M, \quad i=1,2, \ldots, n, \\
& m \leq \lim _{k \rightarrow+\infty} \inf \mu_{i}(k) \leq \lim _{k \rightarrow+\infty} \sup \mu_{i}(k) \leq M, \quad i=1,2, \ldots, n
\end{aligned}
$$

for any solution $x(k)=\left(x_{1}(k), \ldots, x_{n}(k), \mu_{1}(k), \ldots, \mu_{n}(k)\right)$ of system (1.5).

Now, let us consider the first-order difference equation

$$
y(k+1)=A y(k)+B, \quad k=1,2, \ldots,
$$

where $A, B$ are positive constants. Following Lemma 2.1 is a direct corollary of Theorem 6.2 of L. Wang and M. Q. Wang [12, page 125]. 
Lemma 2.2. Assuming that $|A|<1$, for any initial value $y(0)$, there exists a unique solution $y(k)$ of (2.2) which can be expressed as follow:

$$
y(k)=A^{k}\left(y(0)-y^{*}\right)+y^{*},
$$

where $y^{*}=B /(1-A)$. Thus, for any solution $\{y(k)\}$ of system (2.2), one has

$$
\lim _{k \rightarrow+\infty} y(k)=y^{*}
$$

Following comparison theorem of difference equation is Theorem 2.1 of [12, page 241].

Lemma 2.3. Let $k \in N_{k_{0}}^{+}=\left\{k_{0}, k_{0}+1, \ldots, k_{0}+l, \ldots\right\}, r \geq 0$. For any fixed $k, g(k, r)$ is a nondecreasing function with respect to $r$, and for $k \geq k_{0}$, the following inequalities hold:

$$
\begin{aligned}
& y(k+1) \leq g(k, y(k)), \\
& u(k+1) \geq g(k, u(k)) .
\end{aligned}
$$

If $y\left(k_{0}\right) \leq u\left(k_{0}\right)$, then $y(k) \leq u(k)$ for all $k \geq k_{0}$.

Now let us consider the following single species discrete model:

$$
N(k+1)=N(k) \exp \{a(k)-b(k) N(k)\},
$$

where $\{a(k)\}$ and $\{b(k)\}$ are strictly positive sequences of real numbers defined for $k \in N=$ $\{0,1,2, \ldots\}$ and $0<a^{l} \leq a^{u}, 0<b^{l} \leq b^{u}$. Similarly to the proof of Propositions 1 and 3 [13], we can obtain the following.

Lemma 2.4. Any solution of system (2.6) with initial condition $N(0)>0$ satisfies

$$
m \leq \lim _{k \rightarrow+\infty} \inf N(k) \leq \lim _{k \rightarrow+\infty} \sup N(k) \leq M
$$

where

$$
M=\frac{1}{b^{l}} \exp \left\{a^{u}-1\right\}, \quad m=\frac{a^{l}}{b^{u}} \exp \left\{a^{l}-b^{u} M\right\} .
$$

Proposition 2.5. Assume that $\left(H_{1}\right)$ and $\left(H_{2}\right)$ hold, then

$$
\begin{aligned}
& \lim _{k \rightarrow+\infty} \sup x_{i}(k) \leq M_{i}, \quad i=1, \ldots, n, \\
& \lim _{k \rightarrow+\infty} \sup \mu_{i}(k) \leq Q_{i}, \quad i=1, \ldots, n,
\end{aligned}
$$


where

$$
M_{i}=\frac{1}{b_{i i}^{l} \exp \left\{-\left(r_{i}^{u} \tau_{i} / a_{i}^{l}\right)\right\}} \exp \left\{\frac{r_{i}^{u}}{a_{i}^{l}}-1\right\}, \quad Q_{i}=\frac{\left(\beta_{i}^{u}+\gamma_{i}^{u}\right) M_{i}}{\alpha_{i}^{l}}
$$

Proof. Let $x(k)=\left(x_{1}(k), \ldots, x_{n}(k), \mu_{1}(k), \ldots, \mu_{n}(k)\right)$ be any positive solution of system (1.5), from the $i$ th equation of (1.5), we have

$$
x_{i}(k+1) \leq x_{i}(k) \exp \left\{\frac{r_{i}(k)}{a_{i}^{l}}\right\} .
$$

Let $x_{i}(k)=\exp \left\{N_{i}(k)\right\}$, the inequality above is equivalent to

$$
N_{i}(k+1)-N_{i}(k) \leq \frac{r_{i}(k)}{a_{i}^{l}}
$$

Summing both sides of (2.12) from $k-\tau_{i}$ to $k-1$ leads to

$$
\sum_{j=k-\tau_{i}}^{k-1}\left(N_{i}(j+1)-N_{i}(j)\right) \leq \sum_{j=k-\tau_{i}}^{k-1} \frac{r_{i}(j)}{a_{i}^{l}} \leq \frac{r_{i}^{u}}{a_{i}^{l}} \tau_{i}
$$

and so,

$$
N_{i}\left(k-\tau_{i}\right) \geq N_{i}(k)-\frac{r_{i}^{u} \tau_{i}}{a_{i}^{l}}
$$

therefore,

$$
x_{i}\left(k-\tau_{i}\right) \geq x_{i}(k) \exp \left\{-\frac{r_{i}^{u} \tau_{i}}{a_{i}^{l}}\right\}
$$

Substituting (2.15) to the $i$ th equation of (1.5) leads to

$$
x_{i}(k+1) \leq x_{i}(k) \exp \left\{\frac{r_{i}(k)}{a_{i}^{l}}-b_{i i}(k) \exp \left\{-\frac{r_{i}^{u} \tau_{i}}{a_{i}^{l}}\right\} x_{i}(k)\right\} \text {. }
$$

By applying Lemmas 2.3 and 2.4, it immediately follows that

$$
\lim _{k \rightarrow+\infty} \sup x_{i}(k) \leq \frac{1}{b_{i i}^{l} \exp \left\{-r_{i}^{u} \tau_{i} / a_{i}^{l}\right\}} \exp \left\{\frac{r_{i}^{u}}{a_{i}^{l}}-1\right\}:=M_{i}
$$


For any positive constant $\varepsilon$ small enough, it follows from (2.17) that there exists enough large $K_{0}$ such that

$$
x_{i}(k) \leq M_{i}+\varepsilon, \quad i=1, \ldots, n, \quad \forall k \geq K_{0} .
$$

From the $n+i$ th equation of the system (1.5) and (2.18), we can obtain

$$
\Delta \mu_{i}(k) \leq-\alpha_{i}(k) \mu_{i}(k)+\left(\beta_{i}(k)+\gamma_{i}(k)\right)\left(M_{i}+\varepsilon\right),
$$

for all $k \geq K_{0}+\max \left\{\sigma_{i}, i=1, \ldots, n.\right\}$. And so,

$$
\mu_{i}(k+1) \leq\left(1-\alpha_{i}^{l}\right) \mu_{i}(k)+\left(\beta_{i}^{u}+\gamma_{i}^{u}\right)\left(M_{i}+\varepsilon\right)
$$

for all $k \geq K_{0}+\max \left\{\sigma_{i}, i=1,2, \ldots, n.\right\}$. Noticing that $0<1-\alpha_{i}^{l}<1(i=1,2, \ldots, n)$, by applying Lemmas 2.2 and 2.3, it follows from (2.20) that

$$
\lim _{k \rightarrow+\infty} \sup \mu_{i}(k) \leq \frac{\left(\beta_{i}^{u}+\gamma_{i}^{u}\right)\left(M_{i}+\varepsilon\right)}{\alpha_{i}^{l}}
$$

Setting $\varepsilon \rightarrow 0$ in the inequality above leads to

$$
\lim _{k \rightarrow+\infty} \sup \mu_{i}(k) \leq \frac{\left(\beta_{i}^{u}+\gamma_{i}^{u}\right) M_{i}}{\alpha_{i}^{l}}:=Q_{i}
$$

This completes the proof of Proposition 2.5.

Now we are in the position of stating the permanence of system (1.5).

Theorem 2.6. Assume that $\left(\mathrm{H}_{1}\right)$ and $\left(\mathrm{H}_{2}\right)$ hold, assume further that

$$
\frac{r_{i}^{l}}{M_{i}+a_{i}^{u}}-\sum_{j=1, j \neq i}^{n} b_{i j}^{u} M_{j}-c_{i}^{u}-\left(d_{i}^{u}+e_{i}^{u}\right) Q_{i}>0, \quad i=1,2, \ldots, n,
$$

then system (1.5) is permanent.

Proof. By applying Proposition 2.5, we see that to end the proof of Theorem 2.6, it is enough to show that under the conditions of Theorem 2.6,

$$
\begin{aligned}
& \lim _{k \rightarrow+\infty} \inf x_{i}(k) \geq m_{i}, \quad i=1,2, \ldots, n, \\
& \lim _{k \rightarrow+\infty} \inf \mu_{i}(k) \geq q_{i}, \quad i=1,2, \ldots, n .
\end{aligned}
$$


From Proposition 2.5, for all $\varepsilon>0$, there exists a $K_{1}>0, K_{1} \in N$, for all $k>K_{1}$,

$$
x_{i}(k) \leq M_{i}+\varepsilon ; \quad \mu_{i}(k) \leq Q_{i}+\varepsilon, \quad i=1,2, \ldots, n
$$

From the $i$ th equation of system (1.5) and (2.25), we have

$$
x_{i}(k+1) \geq x_{i}(k) \exp \left\{A^{\varepsilon}(k)\right\}, \quad \forall k>K_{1}+\tau,
$$

where

$$
A^{\varepsilon}(k)=\frac{r_{i}(k)}{\left(M_{i}+\varepsilon\right)+a_{i}^{u}}-\sum_{j=1}^{n} b_{i j}(k)\left(M_{j}+\varepsilon\right)-c_{i}(k)-\left(d_{i}(k)+e_{i}(k)\right)\left(Q_{i}+\varepsilon\right) .
$$

Let $x_{i}(k)=\exp \left\{N_{i}(k)\right\}$, the inequality above is equivalent to

$$
N_{i}(k+1)-N_{i}(k) \geq A^{\varepsilon}(k) .
$$

Summing both sides of (2.28) from $k-\tau_{i}$ to $k-1$ leads to

$$
\sum_{j=k-\tau_{i}}^{k-1}\left(N_{i}(j+1)-N_{i}(j)\right) \geq\left(A^{\varepsilon}\right)^{l} \tau_{i}
$$

and so,

$$
N_{i}\left(k-\tau_{i}\right) \leq N_{i}(k)-\left(A^{\varepsilon}\right)^{l} \tau_{i}
$$

where

$$
\left(A^{\varepsilon}\right)^{l}=\frac{r_{i}^{l}}{\left(M_{i}+\varepsilon\right)+a_{i}^{u}}-\sum_{j=1}^{n} b_{i j}^{u}\left(M_{j}+\varepsilon\right)-c_{i}^{u}-\left(d_{i}^{u}+e_{i}^{u}\right)\left(Q_{i}+\varepsilon\right) .
$$

Therefore,

$$
x_{i}\left(k-\tau_{i}\right) \leq x_{i}(k) \exp \left\{-\left(A^{\varepsilon}\right)^{l} \tau_{i}\right\} .
$$


Substituting (2.32) to the $i$ th equation of (1.5) leads to

$$
\begin{gathered}
x_{i}(k+1) \geq x_{i}(k) \exp \left\{\frac{r_{i}(k)}{\left(M_{i}+\varepsilon\right)+a_{i}^{u}}-\sum_{j=1, j \neq i}^{n} b_{i j}(k)\left(M_{j}+\varepsilon\right)-c_{i}(k)\right. \\
\left.-b_{i i}(k) \exp \left\{-\left(A^{\varepsilon}\right)^{l} \tau_{i}\right\} x_{i}(k)-\left(d_{i}(k)+e_{i}(k)\right)\left(Q_{i}+\varepsilon\right)\right\} \\
=x_{i}(k) \exp \left\{B^{\varepsilon}(k)-b_{i i}(k) \exp \left\{-\left(A^{\varepsilon}\right)^{l} \tau_{i}\right\} x_{i}(k)\right\}
\end{gathered}
$$

for all $k>K_{1}+\tau$, where

$$
B^{\varepsilon}(k)=\frac{r_{i}(k)}{\left(M_{i}+\varepsilon\right)+a_{i}^{u}}-\sum_{j=1, j \neq i}^{n} b_{i j}(k)\left(M_{j}+\varepsilon\right)-c_{i}(k)-\left(d_{i}(k)+e_{i}(k)\right)\left(Q_{i}+\varepsilon\right) .
$$

Condition (2.23) shows that Lemma 2.4 could be apply to (2.33), and so, by applying Lemmas 2.3 and 2.4 , it immediately follows that

$$
\lim _{k \rightarrow+\infty} \inf x_{i}(k) \geq \frac{\left(B^{\varepsilon}\right)^{l}}{b_{i i}^{u} \exp \left\{-\left(A^{\varepsilon}\right)^{l} \tau_{i}\right\}} \exp \left\{\left(B^{\varepsilon}\right)^{l}-b_{i i}^{u} \exp \left\{-\left(A^{\varepsilon}\right)^{l} \tau_{i}\right\} M_{i}\right\}
$$

where

$$
\left(B^{\varepsilon}\right)^{l}=\frac{r_{i}^{l}}{\left(M_{i}+\varepsilon\right)+a_{i}^{u}}-\sum_{j=1, j \neq i}^{n} b_{i j}^{u}\left(M_{j}+\varepsilon\right)-c_{i}^{u}-\left(d_{i}^{u}+e_{i}^{u}\right)\left(Q_{i}+\varepsilon\right)
$$

Setting $\varepsilon \rightarrow 0$ in (2.35) leads to

$$
\lim _{k \rightarrow+\infty} \inf x_{i}(k) \geq \frac{\left(B^{0}\right)^{l}}{b_{i i}^{u} \exp \left\{-\left(A^{0}\right)^{l} \tau_{i}\right\}} \exp \left\{\left(B^{0}\right)^{l}-b_{i i}^{u} \exp \left\{-\left(A^{0}\right)^{l} \tau_{i}\right\} M_{i}\right\},
$$

where

$$
\begin{aligned}
& \left(A^{0}\right)^{l}=\frac{r_{i}^{l}}{M_{i}+a_{i}^{u}}-\sum_{j=1}^{n} b_{i j}^{u} M_{j}-c_{i}^{u}-\left(d_{i}^{u}+e_{i}^{u}\right) Q_{i} \\
& \left(B^{0}\right)^{l}=\frac{r_{i}^{l}}{M_{i}+a_{i}^{u}}-\sum_{j=1, j \neq i}^{n} b_{i j}^{u} M_{j}-c_{i}^{u}-\left(d_{i}^{u}+e_{i}^{u}\right) Q_{i} .
\end{aligned}
$$


For any positive constant $\varepsilon$ small enough, it follows from (2.37) that there exists enough large $K_{2}$ such that

$$
x_{i}(k) \geq m_{i}-\varepsilon, \quad i=1, \ldots, n, \quad \forall k \geq K_{2} .
$$

From the $n+i$ th equation of the system (1.5) and (2.39), we can obtain

$$
\Delta \mu_{i}(k) \geq-\alpha_{i}(k) \mu_{i}(k)+\left(\beta_{i}(k)+\gamma_{i}(k)\right)\left(m_{i}-\varepsilon\right),
$$

for all $k \geq K_{2}+\max \left\{\sigma_{i}, i=1, \ldots, n\right\}$. And so,

$$
\mu_{i}(k+1) \geq\left(1-\alpha_{i}^{u}\right) \mu_{i}(k)+\left(\beta_{i}^{l}+\gamma_{i}^{l}\right)\left(m_{i}-\varepsilon\right)
$$

for all $k \geq K_{2}+\max \left\{\sigma_{i}, i=1,2, \ldots, n\right\}$. Noticing that $0<1-\alpha_{i}^{u}<1(i=1,2, \ldots, n)$, by applying Lemmas 2.2 and 2.3, it follows from (2.41) that

$$
\lim _{k \rightarrow+\infty} \inf \mu_{i}(k) \geq \frac{\left(\beta_{i}^{l}+\gamma_{i}^{l}\right)\left(m_{i}-\varepsilon\right)}{\alpha_{i}^{u}}
$$

Setting $\varepsilon \rightarrow 0$ in the inequality above leads to

$$
\lim _{k \rightarrow+\infty} \inf \mu_{i}(k) \geq \frac{\left(\beta_{i}^{l}+\gamma_{i}^{l}\right) m_{i}}{\alpha_{i}^{u}}
$$

This ends the proof of Theorem 2.6.

Now let us consider the following discrete $N$-species Schoener competition system with time delays:

$$
x_{i}(k+1)=x_{i}(k) \exp \left\{\frac{r_{i}(k)}{x_{i}\left(k-\tau_{i}\right)+a_{i}(k)}-\sum_{j=1}^{n} b_{i j}(k) x_{j}\left(k-\tau_{j}\right)-c_{i}(k)\right\}
$$

where $x_{i}(k)(i=1, \ldots, n)$ is the density of species $X_{i}$. Obviously, system (2.44) is the generalization of system (1.5). From the previous proof, we can immediately obtain the following theorem.

Theorem 2.7. Assume that $\left(H_{1}\right)$ and $\left(H_{2}\right)$ hold, assume further that

$$
\frac{r_{i}^{l}}{M_{i}+a_{i}^{u}}-\sum_{j=1, j \neq i}^{n} b_{i j}^{u} M_{j}-c_{i}^{u}>0, \quad i=1,2, \ldots, n,
$$

then system (2.44) is permanent. 


\section{Acknowledgments}

This work is supported by the Foundation of Education, Department of Fujian Province (JA05204), and the Foundation of Science and Technology, Department of Fujian Province (2005K027).

\section{References}

[1] L. Chen, X. Song, and Z. Lu, Mathematical Models and Methods in Ecology, Sichuan Science and Technology Press, Chengdu, China, 2003.

[2] R. M. May, Theoretical Ecology, Principles and Applications, Sounders, Philadelphia, Pa, USA, 1976.

[3] R. P. Agarwal, Difference Equations and Inequalities: Theory, Methods, and Applications, vol. 228 of Monographs and Textbooks in Pure and Applied Mathematics, Marcel Dekker, New York, NY, USA, 2nd edition, 2000.

[4] J. D. Murry, Mathematical Biology, Springer, New York, NY, USA, 1989.

[5] W. Wang and Z. Lu, "Global stability of discrete models of Lotka-Volterra type," Nonlinear Analysis: Theory, Methods E Applications, vol. 35, no. 7, pp. 1019-1030, 1999.

[6] L. Bai, M. Fan, and K. Wang, "Existence of positive periodic solution for difference equations of a cooperative system," Journal of Biomathematics, vol. 19, no. 3, pp. 271-279, 2004 (Chinese).

[7] H.-F. Huo and W.-T. Li, "Positive periodic solutions of a class of delay differential system with feedback control," Applied Mathematics and Computation, vol. 148, no. 1, pp. 35-46, 2004.

[8] F. D. Chen, X. X. Chen, J. D. Cao, and A. P. Chen, "Positive periodic solutions of a class of nonautonomous single species population model with delays and feedback control," Acta Mathematica Sinica, vol. 21, no. 6, pp. 1319-1336, 2005.

[9] F. Chen, "Positive periodic solutions of neutral Lotka-Volterra system with feedback control," Applied Mathematics and Computation, vol. 162, no. 3, pp. 1279-1302, 2005.

[10] F. Chen, "Permanence in nonautonomous multi-species predator-prey system with feedback controls," Applied Mathematics and Computation, vol. 173, no. 2, pp. 694-709, 2006.

[11] F. Chen, "Permanence of a discrete $N$-species cooperation system with time delays and feedback controls," Applied Mathematics and Computation, vol. 186, no. 1, pp. 23-29, 2007.

[12] L. Wang and M. Q. Wang, Ordinary Difference Equation, Xinjiang University Press, Xinjiang, China, 1991.

[13] F. Chen, "Permanence and global attractivity of a discrete multispecies Lotka-Volterra competition predator-prey systems," Applied Mathematics and Computation, vol. 182, no. 1, pp. 3-12, 2006. 